\title{
EL CAPITAL SOCIAL, UN VACÍO EN LA COMISIÓN DE LOS DETERMINANTES SOCIALES DE LA SALUD
}

\section{THE SOCIAL CAPITAL,A MISSED SUBJECT IN THE COMMISSION OF THE SOCIAL DETERMINANTS OF HEALTH}

\author{
León-Manco Roberto Antonio. ${ }^{1}$ \\ Departamento Académico de Odontología Social de la Facultad de Estomatología de la Universidad Peruana Cayetano \\ Heredia, Coordinador de la Sección de Intervención Comunitaria. Perú. ${ }^{1}$ \\ roberto.leon@upch.pe
}

\begin{abstract}
Resumen
En el año 2011 se llevó a cabo la Primera Conferencia de Determinantes Sociales de la salud organizada por la Organización Mundial de la Salud, adoptando un paradigma en salud pública: mejorar los determinantes sociales de la salud para lograr disminuir las brechas de inequidad en salud entre las personas. Sin embargo, han existido muchos críticos a esta posición, indicando que es una visión sesgada de la realidad la cual no debería circunscribirse a políticas públicas en temas de educación, empleo, medioambiente, etc, deben abordar también fenómenos como el trabajo infantil, el terrorismo, violencia familiar, etc. A pesar de ello, ambos enfoques consideran que la construcción de redes sociales generadoras de lazos de intercambio de recursos, refiriéndose al capital social, son de alta relevancia para la mejora de estos determinantes. Por ello, la presente revisión intenta evidenciar el rol del capital social dentro de la teoría de los determinantes sociales de la salud.
\end{abstract}

Palabras clave: Participación comunitaria, participación social, política de salud, redes comunitarias, determinantes sociales de la salud.

\begin{abstract}
In 2011, the First Conference of Social Determinants of Health organized by the World Health Organization was held, adopting a paradigm in public health, to improve the social determinants of health in order to reduce the inequity gaps in health. Between people. However, there have been many critics to this position, indicating that it is a biased vision of reality which should not be limited to public policies on issues of education, employment, environment, etc., should also address phenomena such as child labor, terrorism, family violence, etc. In spite of this, both approaches consider that the construction of social networks that generate ties of exchange of resources, referring to social capital, are highly relevant for the improvement of these determinants. Therefore, the present review attempts to demonstrate the role of social capital within the theory of social determinants of health.
\end{abstract}

Key words: Community participation, social participation, health policy, community networks, social determinants of health.

\section{INTRODUCCIÓN}

La salud pública tiene un nuevo paradigma, los Determinantes Sociales de la Salud (DSS), los cuales según la Organización Mundial de la Salud (OMS) están definidos como el "conjunto de las condiciones sociales en las cuales la gente vive y trabaja". ${ }^{1}$ Esta nueva corriente inicia en el año 2005 con la formación de la Comisión de los Determinantes Sociales de la Salud (CDSS) encabezada por el Dr. Michael Marmot. El informe elaborado por esta comisión comienza con la descripción de las diferentes esperanzas de vida en años de nacidos en distintos países, afirmando que las personas más pobres son aquellas que morirán o enfermarán con mayor frecuencia. La idea principal de este trabajo va por esa línea, la de identificar las desigualdades en salud y la necesidad de eliminar las inequidades, a lo cual denomina "gradiente social de salud", que no es más que la relación directa entre altos índices de morbilidad y mortalidad y bajo nivel socioeconómico. ${ }^{2}$ Sin embargo, no es la primera 
vez que la OMS aborda los temas sociales como causas de enfermedades,: desde su formación la OMS ha definido a la salud como el estado completo bienestar físico, mental y social, pero en su misma acta de constitución se aborda el tema de la importancia de las redes sociales, señalando que la salud depende tanto de la cooperación individual como del Estado. $^{3}$

Son muchos los momentos importantes para la OMS, pero siempre son rememorados al momento de hablar de los DSS dos de ellos: la Primera Conferencia de Atención Primaria de Salud con la Declaración de Alma Ata en 1978, y la primera Conferencia de Promoción de la Salud con la Carta de Ottawa en 1986. Ambos eventos también abordaron la idea de la importancia de las redes sociales o de apoyo para mejorar los logros en salud, Alma Ata promoviendo la participación colectiva en la planificación de salud, y Ottawa adicionando la relevancia del trabajo recíproco y la necesidad de cuidarse los unos a los otros. ${ }^{4,5}$ En este mismo sentido, la Declaración de Río, como producto de la Primera Conferencia de Determinantes Sociales de la Salud realizaba en el año 2011, exhorta a la participación entre los diversos sectores públicos y privados para solucionar los problemas de salud. ${ }^{6}$ En este sentido, la importancia de las redes sociales para alcanzar la transformación real de una población está esclarecido, ${ }^{7}$ pero se evidencia que la CDSS no aborda completamente el tema, y se limita a mencionarlo en sus informes, a omitirlo en algunos casos, o tímidamente colocarlo en algún esquema, tal es el caso del último informe del 2011, donde retoman el tema de redes sociales desde el punto de vista del capital social, mencionándolo en un único esquema indicando que éste da el dinamismo entre los determinantes estructurales e intermedios. ${ }^{8}$

El capital, en general, puede entenderse como conjunto de recursos que facilitan los procesos de desarrollo, ayudan a entender la problemática de las comunidades y países en el objetivo de alcanzar el cubrimiento de sus necesidades. Esta definición permite el abordaje global de lo que es el mundo social, en el cual se evidencia la pobreza, exclusión y conflicto, donde no se debe analizar únicamente estos problemas, sino a su vez las capacidades de la población para la superación de los mismos, los cuales son sus capitales. ${ }^{9}$

Pero la idea de capital social es reciente en las ciencias sociales, y más aún en salud. ${ }^{10}$ Tiene sus orígenes hacia finales de los años 90s, cuando el mismo Banco Mundial generó duras críticas al Consenso de Washington lideradas por su entonces Economista en Jefe Joseph Stiglitz. La evidencia indicaba que las reformas económicas no eran adecuadas y que los países que habían seguido al pie de la letra la "receta económica" no lograron el bienestar esperado, mientras que mejoraron quienes no lo hicieron. La pobreza en 1990 llegó a $48.3 \%$ en América Latina, y al año 1997 sólo descendió a $43.5 \%$, por lo que era necesario una segunda generación de reformas orientadas a cuestiones sociales, y ya no basadas en la estabilización y liberalización. ${ }^{11}$
La posición más radical en contra del Consenso de Washington la tiene J. Stiglitz, quien en una conferencia el 7 de enero de 1998 en el World Institute for Development Economics Research afirmó que; el consenso defendía políticas incompletas, y que su objetivo de crecimiento económico no era suficiente, J.Stiglitz propuso la regulación y supervisión del sector financiero, defensa de la competitividad, fomento de la transferencia de técnicas foráneas, mejorar la administración de justicia, fortalecimiento del estado de derecho, descentralización del poder, fortalecer los gobiernos locales, promover agrupaciones políticas más representativas, e incremento del capital humano. ${ }^{12-15}$ Frente al fracaso del consenso, el Banco Mundial generó nuevas estrategias para la superación de la pobreza, naciendo de esta manera el "capital social" entendido como "el conjunto de recursos obtenidos como consecuencia de las relaciones de unos con otros teniendo de base la confianza, cooperación y reciprocidad. ${ }^{16} \mathrm{~A}$ partir de ello el capital social ha sido materia de estudio no sólo en temas de superación de la pobreza, en la actualidad ha tomado mucha importancia en el área de la salud. ${ }^{17-19}$

\section{NUEVAS ESTRATEGIAS EN PROGRAMAS DE SALUD.}

Según Woolcock y Narayan ${ }^{17}$ el capital social se clasifica en tres tipos en función de la diferencia de niveles de poder y la ubicación geográfica: capital social de unión: destinado a los lazos más íntimos y próximos de los individuos donde existen similares niveles de poder y están delimitados geográficamente, siendo estas características sus principales limitaciones por el impedimento de conseguir recursos distintos a los disponibles en una comunidad. Son las uniones familiares, amistades y en algunos casos lazos comunitarios. ${ }^{20}$ Capital social de puente: capital social referido a lazos entre personas u organizaciones comunitarias con similares niveles de poder pero ubicadas geográficamente en lugares distintos. La intensidad de los lazos de este capital social es menor en comparación con el de unión, sin embargo son durables en el tiempo. Ejemplo de este tipo de capital social son: asociaciones de agricultores, comités de salud, comités de autodefensa, etc. Este tipo de capital social en muchos casos se refiere a organizaciones comunitarias. Capital social de escalera: se refiere a lazos entre personas, organizaciones comunitarias, instituciones estatales y privadas donde se observan distintos niveles de poder sin importar la ubicación geográfica. Ejemplos de este tipo de capital son las relaciones que se presentan entre comunidades e instituciones del estado, empresas privadas y organizaciones no gubernamentales. Este tipo de relaciones facilitan el acceso a otros recursos de nivel político y social. Los tipos de capital social mencionados anteriormente pueden facilitar la obtención de otros recursos y la satisfacción de necesidades del individuo. Además, es obvio que cada uno de estos tipos de capital social tiene diferentes oportunidades y limitaciones. Por su lado, el capital social de unión permite la obtención rápida de recursos en 
la misma localidad, y brinda mayor sentido de pertenencia, sin embargo demanda mayores compromisos, reciprocidad y controles sociales. ${ }^{21}$ Con el capital social de puente y escalera el individuo se siente en la incertidumbre por la inseguridad que significa el cumplimiento de los compromisos poniendo en riesgo la obtención de los recursos, por ello, siempre estará en duda al momento de invertir en la generación de estos tipos de capital social. Esta debilidad se evidencia en la poca institucionalidad de las organizaciones de la comunidad, en la desintegración de los movimientos sociales, la poca participación de las personas, y el favoritismo por la creación de lazos de unión dejando de lado la importancia de los lazos con las organizaciones de la comunidad, estatales y foráneas. ${ }^{16}$ Pero el capital social de puente y escalera permiten el acceso a recursos y niveles de satisfacción que el capital social de unión nunca podrá ofrecer. Sólo por medio del capital social de puente se pueden conseguir cambios a nivel político en la distribución de los recursos, además de influenciar en las organizaciones comunitarias que controlan estos recursos. A sí mismo, sólo mediante el capital social de escalera se pueden conseguir recursos de instituciones estatales, no estatales y foráneas; y a través del capital social de puente y escalera se puede participar en la administración pública para el diseño de las políticas públicas. ${ }^{16}$

Las asociaciones del capital social con otras variables han sido evaluadas en diversos ámbitos, tales como educación escolar, criminología, migración, realización laboral, democracia y gobernabilidad. Particularmente, se ha analizado su posible impacto en el desarrollo socio-económico y en la superación de la pobreza. Pero las investigaciones respecto a la asociación entre capital social y salud son escasas, más aún en el contexto latinoamericano. ${ }^{22}$ En general, todos los trabajos de investigación toman como punto inicial la definición de Putnam al indicar que el capital social es la "goma invisible" que une a una sociedad. A partir de ello, Kawachi et al. intentaron probar los daños físicos resultando de una inadecuada cohesión social, llegando a concluir que un alto nivel de salud depende de la distribución equitativa de los ingresos. ${ }^{23}$

Los estudios al respecto del capital social han revelado que existe relación directa entre éste y un adecuado funcionamiento de las instituciones estatales y privadas. Con relaciones de este tipo establecidas en la sociedad, los ciudadanos comienzan a confiar en estas instituciones de la cual forma parte el sistema de salud. Teniendo presente esta visión es obvio que una sociedad más participativa y organizada tendrá mayor capital social acumulado, por consiguiente, existirá influencia en el logro de los objetivos del sistema de salud. ${ }^{24}$

La evidencia científica confirma que las comunidades con mayor capital social presentan una mayor expectativa de vida y una mejor percepción de su salud, así como menores tasas de morbilidad y mortalidad por causas generales y específicas. ${ }^{23-25}$ Pese a esta evidencia, existen algunos estudios que no muestran estas asociaciones indicando que los resultados pudieron estar influenciados por otros factores que las investigaciones no han tomado en cuenta. ${ }^{26}$ Según Morgan y Swann, el capital social puede influenciar en la salud de las personas a través de varios medios: desde un nivel macro de análisis, el capital social puede facilitar el acceso a un servicio de salud. Cuanto mejor es la red social entre y cada uno de los grupos proveedores de salud, se garantiza un eficiente y eficaz servicio de salud. La comunidad y las organizaciones voluntarias juegan un rol importante en brindar servicios a los pacientes tanto de países en desarrollo como en los industrializados. Desde un nivel meso de análisis, el capital social puede mejorar la salud a través del impulso y cambio de las normas sociales. Una sociedad más cohesionada, con un sentimiento fuerte de identidad de grupo tiende a buscar un bienestar común, por ejemplo: eliminar la contaminación, propuestas para detener el hábito de fumar, incentivar adecuados hábitos en salud bucal, etc. ${ }^{27}$ Desde una perspectiva micro, las interacciones sociales intensas ofrecen un canal privilegiado para la transmisión de información y búsquedas de experiencias pasadas en adecuados hábitos de salud, doctores, fármacos y enfermedades que reducen el costo de información de salud. El capital social puede a su vez impulsar los esfuerzos en el área de la prevención, la cual puede ser efectiva, si es sostenida por redes formales e informales a través de personas que reciben información y medicina. $^{27}$

\section{LA “GOMA INVISIBLE” OLVIDADA EN SALUD.}

Morgan et al. establecieron que el capital social puede mejorar la salud a través del impulso y cambio de las normas sociales, y cuanto mejor es la red social entre y cada uno de los grupos proveedores de salud, más eficiente y eficaz es el servicio de salud. Ellos indicaron que una sociedad más cohesionada tiende a buscar un bienestar común; por ejemplo, eliminar la contaminación, propuestas para detener el hábito de fumar, incentivar adecuados hábitos en salud bucal, etc. ${ }^{27}$ Se debe considerar que no basta con la creación de comités de salud donde las relaciones entre los individuos sean de alta calidad: es necesaria la creación de redes verticales que garanticen un adecuado intercambio de conocimientos entre la población y los profesionales de salud. Se debe reconocer que el establecimiento de relaciones de reciprocidad y confianza tiene como eslabón inicial el interés y la habilidad para establecer relaciones humanas saludables. ${ }^{9}$ Como se mencionó antes, existe evidencia científica ambigua al relacionar el capital social con el estado de salud de las personas, pero sí está claro que es una estrategia adecuada en la implementación de programas para el desarrollo. ${ }^{16} \mathrm{El}$ Instituto de Estudios de Desarrollo (Institute Development Studies) de la Universidad de Sussex indicó en el año 1999 que sus programas de investigación tienen como término unificador al capital social: de igual manera, frente a la problemática de gobernabilidad y rol de la sociedad civil, el Banco Mundial en su Reporte de Desarrollo Mundial (World 
Development Report) del año 1999 indicó que el capital social es un pilar para la superación de este problema. ${ }^{28}$ Así mismo, para el Banco Interamericano de Desarrollo, el capital social es una variable indispensable en el diseño y evaluación de sus proyectos. ${ }^{9}$ Por su lado la Organización Mundial de la Salud, como se mencionó, en el año 1978 emite la Declaración de Alma Ata la cual propone como estrategia la Atención Primaria de Salud que consta de la participación activa de las personas en el desarrollo de los programas de salud. ${ }^{4}$ Posteriormente en la Declaración de Yakarta (1999) se expresa que la promoción de la salud debe estar basada en el control de las personas sobre sus DSS y la construcción de capital social, ${ }^{29}$ de forma indirecta la Declaración de México (2000) indica la necesidad de reforzar y ampliar los vínculos de asociación en beneficio de la salud: en la misma línea la Carta de Bangkok (2005) y el Compromiso de Chile (2006) enfatizan en la importancia de la participación de los individuos en las estrategias de promoción de la salud, ${ }^{20}$ todo esto sustenta el hecho de introducir al capital social en los programas de salud. El fomentar la generación de capital social es esencial para todo proceso de desarrollo sostenible: esta generación se logra mediante el incentivo del cambio social, el empoderamiento y la contribución a la creación de lazos verticales entre individuos y organizaciones. ${ }^{9}$ Es solo por medio de la participación con organizaciones que se puedeN lograr cambios a nivel estructural que determinen una adecuada distribución de recursos y a su vez influenciar en las instituciones controladoras de estos activos, ${ }^{16}$ los cuales tienen repercusión en indicadores y prácticas saludables. ${ }^{30,31}$

Es momento de expandir los horizontes establecidos y comprender a la salud no como un estado de bienestar físico, mental y social: por el contrario, debe ser entendida con la visión de desarrollo que tiene Amartya Sen que muy fácilmente es adaptable: como un proceso de expansión de libertades para el disfrute del individuo ${ }^{32}$ tendiendo a las libertades como las oportunidades que las personas gozan para lograr sus objetivos, y estas oportunidades deben ser derechos. No se pueden diseñar programas de salud que tengan como finalidad única instalar mejorar ciertos aspectos de la vida, si en la población objetivo existen problemas mayores como la violencia acumulada, la explotación infantil, o procesos históricos que quiebran sociedades desde hace muchos años. En ese sentido, al tomar este nuevo punto de vista, no es posible concebir una sociedad saludable sin antes pensar en la construcción de una sociedad solidaria, en la cual los individuos forjen en conjunto objetivos comunes, trabajen integradamente y utilicen los preceptos del capital social para ello: confianza, cooperación y reciprocidad. Por ello, cuando se analizan los determinantes sociales de la salud se pueden hacer desde una visión de los determinantes a nivel individual, o desde la perspectiva estructural. El primer enfoque es el más común y es evidenciado en la actualidad, como si una entrevista o encuesta pueda dar una idea de la condición de los determinantes, en ese mismo sentido, la comprensión del capital social como un bien individual es errado. Por ello, para comprender a los mismos DSS, es necesaria la visión estructural, y en ese análisis es indispensable la comprensión de capital social. Este no debe ser más la pretensión de entender que las partes unidas constituyen un todo: al contrario, las partes se integran y modifican por el todo, es decir, necesitamos comprender como estas diferencias de recursos, poder, acceso a información, etc. entre los sujetos como producto de sus relaciones sociales están vinculadas a los DSS, e indirectamente al estado de salud de las personas. ${ }^{33}$ Por ello, es momento de implementar nuevas metodologías en los programas de salud que permitan el éxito de los mismos mediante la sostenibilidad, logrando el empoderamiento de las personas y comunidades. Estos programas deben tener como objetivo inicial el mejoramiento de los DSS, los cuales deben ser entendidos en forma integrada y no aisladamente. No deben ser definidos como "las condiciones en las que una persona vive y trabaja": por el contrario, son las circunstancia en la que una población se desarrolla, porque los determinantes de una persona están vinculados a los de otros, en el presente y pasado. La CDSS ha tenido muchos vacíos: uno de ellos es la importancia de las poblaciones a través de la construcción de redes sociales para el logro de objetivos.

Los nuevos tiempos trae consigo nuevas metodologías para los programas de salud pública con visión transdisciplinaria, las cuales deben implementarse mediante una estrategia generadora de capital social.

Conflicto de intereses y financiamiento El autor declara no tener conflicto de intereses, haber cumplido con los requisitos de autoría y haber autofinanciado este artículo.

\section{Referencias}

1 Organización Mundial de la Salud. Determinantes sociales de la salud. Ginebra: Organización Mundial de la Salud; 2008.

2 Organización Mundial de la Salud. Subsanar las desigualdades en una generación. Ginebra: Organización Mundial de la Salud; 2008.

3 Organización Mundial de la Salud. Constitution of the World Health Organization. Ginebra Organización Mundial de la Salud 1946.

4 Organización Mundial de la Salud. Declaración de Alma Ata. Ginebra Organización Mundial de la Salud; 1978.

5 Organización Mundial de la Salud. Carta de Ottawa. Ginebra: Organización Mundial de la Salud; 1986.

6 Organización Mundial de la Salud. Declaración de Río. Ginebra: Organización Mundial de la Salud; 2011.

7 González Guzmán R. La medicina social ante el reporte de la Comisión sobre los Determinantes Sociales de Salud, Organización Mundial de Salud. Medicina Social. 2009; 135-43. 
8 Organización Mundial de la Salud. Closing the gap: Policy intro practice on social determinants of health. Ginebra: Organización Mundial de la Salud; 2011

9 La Rosa L. Políticas de promoción de la salud y capital social. Lima: Litografía Artística S. A; 2002

10 Lip C, Rocabado F. Determinantes sociales de la salud 27 en Perú. Lima: Ministerio de Salud, Universidad Norbert Wiener, Organización Panamericana de la Salud; 2005.

11 Bustelo P. Desarrollo económica: del Consenso al Post- 28 Consenso de Washington y más allá. En: Bustelo P. Estudios en homenaje al profesor Francisco Bustelo. Madrid: Editorial Complutense; 2003.

12 Stiglitz J. More instruments and broader goals: moving 30 towards the post-washington consensus. World Institute for Development Economics Research. Helsinki; 1998.

13 Stiglitz J. El consenso post consenso de Washington. The Initiative for Policy Dialogue, Fundación CIDOB. Barcelo- 32 na; 2004.

14 Birdsall N, Menezes R. Más allá del Consenso de Washing- 33 ton. Nuevo contrato social en América Latina basado en el empleo. Lima: Grupo de Análisis para el Desarrollo; 2006.

15 Diniz E. El Post-consenso de Washington: globalización, estado y gobernabilidad reexaminados. Madrid: Instituto de Estudios Latinoamericanos; 2007.

16 Bebbington A. Estrategias de vida y estrategias de intervención: el capital social y los programas de superación de la pobreza. En: Arriagada I. Aprender de la Experiencia. El capital social en la superación de la pobreza. Santiago de Chile: CEPAL, Organización de las Naciones Unidas; 2005.

17 Woolcoock M, Narayan D. Social capital: implications for development theory, research and policy. The World Bank; 2000: 15(2).

18 Kripper CE, Sapag JC. Capital social y salud en América Latina y el Caribe: una revisión sistemática. Rev Panam Salud Publica. 2009; 25(2):162-70.

19 La Rosa L. Políticas de promoción de la salud y capital social. Lima: Litografía Artística S. A; 2002.

20 Sapag JC, Kawachi I. Capital social y promoción de la salud en América Latina. Revista de Saúde Pública 2007; 41(1): 139-49.

21 Kawachi I, Lochner K, Kennedy B. Long live community. Social capital as public health. American Prospect; 1997; 8(35): 1-6.

22 Labra M. Capital social y consejos de salud en Brasil. ¿Un círculo virtuoso?. Cadernos de Saúde Pública 2002; 18: $47-$ 55.

23 Petrou S, Kupek E. Social capital and its relationship with measures of health status: evidence from the Health Survey for England 2003. Health Economics 2008; 14(1): 127-43.

24 Kawachi I, Kennedy B, Lochner K, Prothrow-Stith D. Social Capital, Income Inequality, and Mortality. American Journal of Public Health 1997; 87(9): 1491-98.

25 Holtgrave DR, Crosby RA. Social capital, poverty, and income inequality as predictors of gonorrhoea, syphilis, chlamydia and AIDS case rates in the United States. Sex Transm Infect 2003; 79: 62-4.

26 Van der Wel K. Social capital and health-a multilevel analysis of 25 administrative districts in Oslo. Norsk Epidemiologi 2007 ; $17(1)$ : $71-8$.

Morgan A, Swann C. Social capital for health: issues of definition, measurement and links to health. London: NHS Health Development Agency; 2004.

Harris J, De Renzio P. Missing link or analytically missing?: the concept of social capital. 1997; 9:919-37.

29 Organización Mundial de la Salud. Declaración de Yakarta. Ginebra: Organización Mundial de la Salud; 1999.

León-Manco RA. Capital social y caries dental. Berlín: Editorial Académica Española; 2012.

31 León-Manco RA. Capital social y lactancia materna exclusiva. Berlín: Editorial Académica Española; 2017.

32 Sen A. Development as freedom. Oxford: Oxford University Press; 2001.

Breilh J. Las tres "S" de la determinación de la vida y el triángulo de la política. Bahía: Centro Brasileiro de Estudios de Saúde; 2010.
Recibido: 10 de enero de 2017.

Aceptado: 17 de febrero de 2017. 
